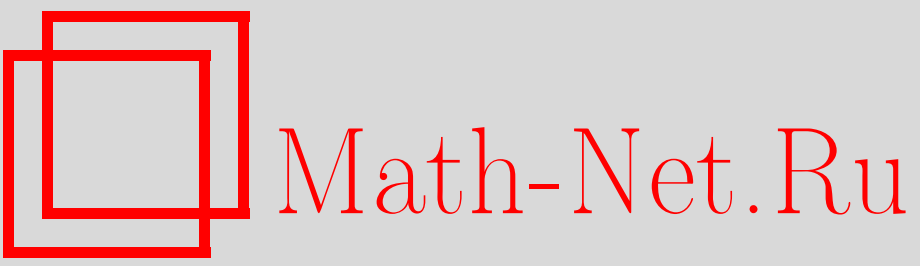

С. К. Голушко, Г. Л. Горынин, А. Г. Горынин, Метод асимптотического расщепления в динамических задачах пространственной теории упругости, Итоги науки и техн. Сер. Соврем. мат. и ее прил. Темат. обз., 2020, том 188, 43-53

DOI: https://doi.org/10.36535/0233-6723-2020-188-43-53

Использование Общероссийского математического портала Math-Net.Ru подразумевает, что вы прочитали и согласны с пользовательским соглашением

http://www.mathnet.ru/rus/agreement

Параметры загрузки:

IP: 35.173 .219 .12

26 апреля 2023 г., 05:04:27 
УДК 517.955.8, 539.3

\title{
МЕТОД АСИМПТОТИЧЕСКОГО РАСЩЕПЛЕНИЯ В ДИНАМИЧЕСКИХ ЗАДАЧАХ ПРОСТРАНСТВЕННОЙ ТЕОРИИ УПРУГОСТИ
}

\author{
(c) 2020 г. $\quad$ С. К. ГОЛУШКО, Г. Л. ГОРЫнИН, А. Г. ГОРЫНИН
}

\begin{abstract}
АннотАция. В работе реализован метод асимптотического расщепления и получены асимптотические решения применительно к динамическим задачам пространственной теории упругости, уравнения которых содержат малый параметр. Возникающие в ходе асимптотического расщепления двумерные и одномерные краевые задачи допускают получение аналитических решений в некоторых частных случаях, а в общем случае они решаются численно при помощи метода коллокаций, метода наименьших квадратов и метода конечных элементов.
\end{abstract}

Ключевые слова: пространственная теория упругости, динамическая задача, метод асимптотического расщепления, метод коллокаций и наименьших квадратов, метод конечных элементов, слоистая балка, свободные колебания.

\section{METHOD OF ASYMPTOTIC SPLITTING IN DYNAMICAL PROBLEMS OF THE SPATIAL THEORY OF ELASTICITY}

\author{
(C) 2020 S. K. GOLUSHKO, G. L. GORYNIN, A. G. GORYNIN
}

\begin{abstract}
In this paper, we apply the method of asymptotic splitting to dynamical problems of the spatial theory of elasticity, whose equations contain a small parameter, and obtain asymptotic solutions. Two-dimensional and one-dimensional boundary-value problems arising in the process of asymptotic splitting allow obtaining analytical solutions in some special cases. In the general case, they can be solved numerically by the collocation method, the method of least squares, and the finite-element method.
\end{abstract}

Keywords and phrases: spatial theory of elasticity, dynamical problem, method of asymptotic splitting, collocation method, method of least squares, finite element method, layered beam, free oscillations.

AMS Subject Classification: 35Q74, 74H10

1. Введение. Пространственная теория упругости, обладающая почти математическим уровнем строгости в постановке своих задач, сложилась в трудах Навье, Пуассона, Коши в начале XIX в., однако с точки зрения методов решения краевых задач для систем уравнений в частных производных теория слабо развита и по нынешний день. Вместо решения динамических задач в математически точной пространственной постановке значительно большее распространение получил подход, связанный с введением тех или иных упрощающих гипотез и к сведению

Работа выполнена при поддержке Российского фонда фундаментальных исследований (проект № 18-29-18029). 
исходной задачи к задачам меньшей размерности. Основные идеи и методы решения задач классической теории упругости на основе введения упрощающих гипотез приведены, в частности, в $[2-5,7,14,15]$.

Сегодня широкое практическое применение находят конструкции со сложной геометрической формой, имеющие слоистую неоднородную структуру. Введение упрощающих гипотез в случае слоистых конструкций является проблематичным по сравнению с классической теорией однородных изотропных конструкций в связи со сложностью возникающего в них напряженно-деформированного состояния, поэтому актуальным является построение логически стройных и математически обоснованных подходов, позволяющих работать с исходной пространственной постановкой задачи. Для задач механики слоистых балок, плит и оболочек характерно наличие малого параметра, связанного с существенной разницей характерных размеров конструкции. В этом случае для решения задач теории упругости применимы асимптотические методы, общая теория которых и их применение в механике твердого тела представлены в $[1,6,16,17]$.

В данной работе для решения динамической задачи линейной теории упругости с малым параметром использован метод асимптотического расщепления (см. [11]), позволяющий свести решение исходной трехмерной задачи к решению последовательности вспомогательных двумерных и одномерных задач. Преимущества данного подхода заключаются в том, что для некоторых частных случаев удается получить точные аналитические решения задачи, а вычислительные затраты на решение двумерных задач значительно ниже чем при решении исходных трехмерных уравнений линейной теории упругости. Для численного решения двумерных краевых задач использованы метод коллокаций и метод наименьших квадратов (см. [8-10, 20, 22]), а также метод конечных элементов, реализованный в пакете с открытым кодом FEniCS Project (см. [19]). Приведено математическое обоснование метода асимптотического расщепления и построение асимптотического решения для динамической краевой задачи с малым параметром, показано применение метода на задаче о собственных колебаниях слоистых балок, представлены численные расчеты и дан сравнительный анализ полученных результатов с известными частными решениями.

2. Математическая постановка задачи. Пусть

$$
\Omega=\left\{(x, y, z):-\frac{1}{2} \leqslant x \leqslant \frac{1}{2},-b_{i} \leqslant y \leqslant b_{i}, 0 \leqslant z \leqslant 1, b_{i} \in \mathbb{R}^{+}, i \in \mathbb{N}\right\}
$$

- замкнутая область со слоистой структурой по переменной $x$ (см. рис. 1), соответствующая некоторому деформируемому телу. Каждому слою соответствует подобласть

$$
\Omega_{i}=\left\{(x, y, z): h_{i-1} \leqslant x \leqslant h_{i},-b_{i} \leqslant y \leqslant b_{i}, 0 \leqslant z \leqslant 1, h_{i} \in\left[-\frac{1}{2} ; \frac{1}{2}\right]\right\},
$$

так что $\Omega=\bigcup_{i=1}^{n} \Omega_{i}$. Таким образом, область $\Omega$ состоит из $n$ слоев, где $i-$ порядковый номер слоя.

В области $\Omega$ рассмотрим систему уравнений для динамической задачи линейной теории упругости с малым параметром $\varepsilon$ :

$$
\begin{gathered}
\frac{\partial\left(\sigma_{\alpha x}\right)_{i}}{\partial x}+\frac{\partial\left(\sigma_{\alpha y}\right)_{i}}{\partial y}+\varepsilon \frac{\partial\left(\sigma_{\alpha z}\right)_{i}}{\partial z}=\rho_{i} \frac{\partial^{2}\left(u_{\alpha}\right)_{i}}{\partial t^{2}}, \quad \alpha, \beta \in(x, y, z), \\
\left(\sigma_{\alpha \beta}\right)_{i}=\lambda_{i} \theta \delta_{\alpha \beta}+2 \mu_{i}\left(e_{\alpha \beta}\right)_{i}, \quad \theta=\sum_{\gamma=1}^{3} e_{\gamma \gamma}, \quad \alpha, \beta \in(x, y, z), \\
\left(e_{\alpha \beta}\right)_{i}=\frac{1}{2}\left(\frac{\partial\left(u_{\alpha}\right)_{i}}{\partial \beta}+\frac{\partial\left(u_{\beta}\right)_{i}}{\partial \alpha}\right), \quad\left(e_{z z}\right)_{i}=\varepsilon \frac{\partial\left(u_{z}\right)_{i}}{\partial z}, \quad \alpha, \beta \in(x, y), \\
\left(e_{x z}\right)_{i}=\frac{1}{2}\left(\varepsilon \frac{\partial\left(u_{x}\right)_{i}}{\partial z}+\frac{\partial\left(u_{z}\right)_{i}}{\partial x}\right), \quad\left(e_{y z}\right)_{i}=\frac{1}{2}\left(\varepsilon \frac{\partial\left(u_{y}\right)_{i}}{\partial z}+\frac{\partial\left(u_{z}\right)_{i}}{\partial y}\right) \\
{\left[\left(\sigma_{\alpha n}\right)\right]_{i}^{j}=0, \quad\left(\sigma_{\alpha n}\right)_{i}=\left(\sigma_{\alpha x}\right)_{i} n_{x}+\left(\sigma_{\alpha y}\right)_{i} n_{y}, \quad\left(u_{\alpha}\right)_{j}=\left(u_{\alpha}\right)_{i} .}
\end{gathered}
$$

Коэффициенты Ламе $\lambda_{i}, \mu_{i}$ и плотность тела $\rho_{i}$-разрывные положительные величины, постоянные в каждом отдельном слое $\Omega_{i}$. Для склейки неизвестных между слоями используется 

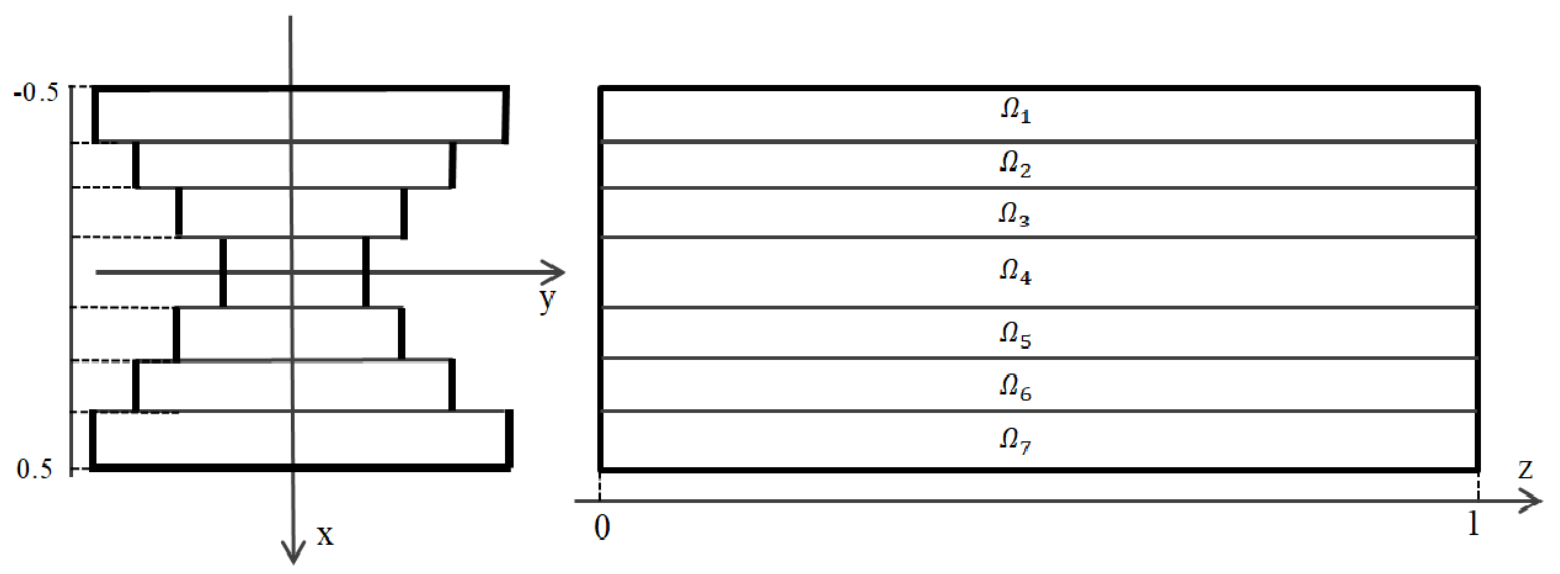

Рис. 1. Область $\Omega$ со слоистой структурой

условие (1d), что означает непрерывность вектора перемещений и контактных напряжений. Система (1) состоит из 15 уравнений на нахождение 15 неизвестных: 6 компонент тензора напряжений $\sigma_{\alpha \beta}, 6$ компонент тензора деформаций $e_{\alpha \beta}$ и 3 компоненты вектора перемещений $u_{\alpha}$. Закон Гука (1b) задает линейную связь между компонентами тензора деформации и тензора напряжений в каждом слое. Уравнения совместности (1c) описывают связь между тензором деформации и вектором перемещений. Характерный размер тела по оси $z$ существенно больше характерных размеров по осям $x, y$.

Краевые условия задаются в напряжениях на границе области $x= \pm 1 / 2, y= \pm b$ :

$$
\left(\sigma_{\alpha x}\right)_{i} n_{x}+\left(\sigma_{\alpha y}\right)_{i} n_{y}=0, \quad x= \pm \frac{1}{2}, \quad y= \pm b .
$$

Задача (1), (2) описывает свободные колебания вдоль оси $x$ тела, ограниченного областью $\Omega$. Для однозначной разрешимости системы (1) необходимо задать недостающие краевые условия на границах $z=0, z=1$ :

$$
\frac{1}{b} \int_{-1 / 2}^{1 / 2} \int_{-b}^{b}\left(u_{x}\right)_{i} d y d x=0, \quad \int_{-1 / 2}^{1 / 2} \int_{-b}^{b} x\left(\sigma_{z z}\right)_{i} d y d x=0, \quad z=0,1 .
$$

Систему уравнений (1), (2) без условий закрепления (3) будем называть полукраевой задачей, так как для построения асимптотического решения целесообразно сначала рассмотреть задачу без части краевых условий.

3. Метод асимптотического расщепления. Основная идея метода асимптотического расщепления (см. [21]) состоит в том, что неизвестные вектор-функции перемещений и тензорфункции напряжений ищутся в виде конечных сумм степеней дифференциальных операторов от некоторой функции $u_{0}(z, t)$, зависящей от продольной координаты $z$. Функция $u_{0}$ выбирается индивидуально для разного типа задач исходя из физических соображений.

Для компонент вектора перемещений на каждом слое $\Omega_{i}$ примем следующие правила аппроксимации:

$$
\begin{gathered}
\left(u_{\beta}\right)_{i}^{(n)}(\bar{r}, t, \varepsilon)=\sum_{k=0}^{n+1}\left(U_{\beta}\right)_{i}^{(2 k)} \frac{\partial^{2 k} u_{0}^{(n)}}{\partial z^{2 k}} \varepsilon^{2 k}, \quad \beta \in(x, y), \\
\left(u_{z}\right)_{i}^{(n)}(\bar{r}, t, \varepsilon)=\sum_{k=0}^{n}\left(U_{z}\right)_{i}^{(2 k+1)} \frac{\partial^{2 k+1} u_{0}^{(n)}}{\partial z^{2 k+1}} \varepsilon^{2 k+1}, \quad u_{0}^{(n)}(z, t)=\frac{1}{F} \sum_{i=1}^{s} \int_{F_{i}}\left(u_{x}\right)_{i}^{(n)}(\bar{r}, t, \varepsilon) d F, \\
\left(U_{x}\right)_{i}^{(0)}=1, \quad\left(U_{y}\right)_{i}^{(0)}=0, \quad\left(U_{z}\right)_{i}^{(1)}=-\left(x-c_{0}\right),
\end{gathered}
$$


где $\left(U_{\alpha}\right)_{i}^{(k)}$ - жесткостные функции вектора перемещений; $\left(u_{\alpha}\right)_{i}^{(n)}(\bar{r}, t, \varepsilon)$ - компоненты вектора перемещений точек $i$-го слоя для асимптотического приближения с номером $n ; u_{0}(z, t)$ - функция, равная среднему перемещению точек поперечного сечения в направлении оси $x ; F$ - площадь поперечного сечения области $\Omega$, перпендикулярного оси $z$.

Аппроксимация для компонент тензора напряжений:

$$
\begin{gathered}
\left(\sigma_{\alpha \beta}\right)_{i}^{(n)}(\bar{r}, t, \varepsilon)=\sum_{k=1}^{n+1}\left(\tau_{\alpha \beta}\right)_{i}^{(2 k)} \frac{\partial^{2 k} u_{0}^{(n)}}{\partial z^{2 k}} \varepsilon^{2 k}, \quad\left(\sigma_{z z}\right)_{i}^{(n)}(\bar{r}, t, \varepsilon)=\sum_{k=1}^{n}\left(\tau_{z z}\right)_{i}^{(2 k)} \frac{\partial^{2 k} u_{0}^{(n)}}{\partial z^{2 k}} \varepsilon^{2 k} \\
\left(\sigma_{\alpha z}\right)_{i}^{(n)}(\bar{r}, t, \varepsilon)=\sum_{k=1}^{n}\left(\tau_{\alpha z}\right)_{i}^{(2 k+1)} \frac{\partial^{2 k+1} u_{0}^{(n)}}{\partial z^{2 k+1}} \varepsilon^{2 k+1}, \quad \alpha, \beta \in(x, y)
\end{gathered}
$$

где $\left(\tau_{\alpha \beta}\right)_{i}^{(k)}(x, y)$ - жесткостные функции тензорного поля напряжений в поперечном сечении области $\Omega$.

Для функции $u_{0}^{(n)}$ положим выполнение следующего уравнения (см. [21]):

$$
\frac{\partial^{2} u_{0}^{(n)}}{\partial t^{2}}+\sum_{k=2}^{n+1} p_{x}^{(2 k)} \frac{\partial^{2 k} u_{0}^{(n)}}{\partial z^{2 k}} \varepsilon^{2 k}=0
$$

где $p_{x}^{(2 k)}$ - некоторые положительные константы.

Связь между жесткостными функциями тензорного поля напряжений и жесткостными функциями векторного поля перемещений следует из подстановки (4), (5) в (1b), (1c) и имеет вид

$$
\begin{gathered}
\left(\tau_{x y}\right)_{i}^{2 k}=\mu_{i}\left(\frac{\partial\left(U_{y}\right)_{i}^{(2 k)}}{\partial x}+\frac{\partial\left(U_{x}\right)_{i}^{(2 k)}}{\partial y}\right), \quad\left(\tau_{z \alpha}\right)_{i}^{2 k+1}=\mu_{i}\left(\frac{\partial\left(U_{z}\right)_{i}^{(2 k+1)}}{\partial \alpha}+\left(U_{\alpha}\right)_{i}^{(2 k)}\right), \quad \alpha \in(x, y), \\
\left(\tau_{z z}\right)_{i}^{2 k}=\left(\lambda_{i}+2 \mu_{i}\right)\left(U_{z}\right)_{i}^{(2 k-1)}+\lambda_{i}\left(\frac{\partial\left(U_{x}\right)_{i}^{(2 k)}}{\partial x}+\frac{\partial\left(U_{y}\right)_{i}^{(2 k)}}{\partial y}\right), \\
\left(\tau_{x x}\right)_{i}^{2 k}=\left(\lambda_{i}+2 \mu_{i}\right) \frac{\partial\left(U_{x}\right)_{i}^{(2 k)}}{\partial x}+\lambda_{i}\left(\left(U_{z}\right)_{i}^{(2 k-1)}+\frac{\partial\left(U_{y}\right)_{i}^{(2 k)}}{\partial y}\right), \\
\left(\tau_{y y}\right)_{i}^{2 k}=\left(\lambda_{i}+2 \mu_{i}\right) \frac{\partial\left(U_{y}\right)_{i}^{(2 k)}}{\partial y}+\lambda_{i}\left(\left(U_{z}\right)_{i}^{(2 k-1)}+\frac{\partial\left(U_{x}\right)_{i}^{(2 k)}}{\partial x}\right) .
\end{gathered}
$$

Жесткостные функции связаны следующими равенствами, вытекающими из закона Гука (1b):

$$
\begin{gathered}
\left(\tau_{z z}\right)_{i}^{(2)}=\nu_{i}\left(\tau_{x x}\right)_{i}^{(2)}+\nu_{i}\left(\tau_{y y}\right)_{i}^{(2)}-E_{i}\left(x-c_{0}\right), \\
\left(\tau_{z z}\right)_{i}^{(4)}=\nu_{i}\left(\tau_{x x}\right)_{i}^{(4)}+\nu_{i}\left(\tau_{y y}\right)_{i}^{(4)}+E_{i}\left(U_{z}\right)_{i}^{(3)}
\end{gathered}
$$

где $E_{i}, \nu_{i}$ - модуль Юнга и коэффициент Пуассона для материала в $i$-м слое соответственно.

3.1. Вспомогательные двумерные краевые задачи. Аппроксимация вектора перемещений $\left(u_{\alpha}\right)_{i}^{(n)}$ и тензора напряжений $\left(\sigma_{\alpha \beta}\right)_{i}^{(n)}$ в виде $(4),(5)$ позволяет свести исходную трехмерную задачу линейной теории упругости к решению $(n+1)$ двумерных краевых задач для нахождения жесткостных функций тензора напряжений $\left(\tau_{\alpha \beta}\right)_{i}^{(n)}$. Выразим частную производную по времени из (6) и подставим в уравнения движения (1a) вместе с формулами (4), (5). Приравняв коэффициенты при степенях малого параметра, получим последовательность систем уравнений в зависимости от жесткостного номера $k$, меняющегося от 1 до $n+1$. Приведем первые три из них. 
При $k=1$ :

$$
\left\{\begin{array}{l}
\frac{\partial\left(\tau_{\alpha x}\right)_{i}^{(2)}}{\partial x}+\frac{\partial\left(\tau_{\alpha y}\right)_{i}^{(2)}}{\partial y}=0 \\
\frac{\partial\left(\tau_{z x}\right)_{i}^{(3)}}{\partial x}+\frac{\partial\left(\tau_{z y}\right)_{i}^{(3)}}{\partial y}+\left(\tau_{z z}\right)_{i}^{(2)}=0
\end{array}\right.
$$

при $k=2$ :

при $k=3$ :

$$
\left\{\begin{array}{l}
\frac{\partial\left(\tau_{x x}\right)_{i}^{(4)}}{\partial x}+\frac{\partial\left(\tau_{x y}\right)_{i}^{(4)}}{\partial y}+\left(\tau_{x z}\right)_{i}^{(3)}+\rho_{i} \frac{G_{x z}^{(3)}}{M}=0 \\
\frac{\partial\left(\tau_{x y}\right)_{i}^{(4)}}{\partial x}+\frac{\partial\left(\tau_{y y}\right)_{i}^{(4)}}{\partial y}+\left(\tau_{y z}\right)_{i}^{(3)}=0 \\
\frac{\partial\left(\tau_{z x}\right)_{i}^{(5)}}{\partial x}+\frac{\partial\left(\tau_{z y}\right)_{i}^{(5)}}{\partial y}+\left(\tau_{z z}\right)_{i}^{(4)}-\rho_{i} \frac{G_{x z}^{(3)}}{M}\left(x-c_{0}\right)=0
\end{array}\right.
$$

$$
\left\{\begin{array}{l}
\frac{\partial\left(\tau_{x x}\right)_{i}^{(6)}}{\partial x}+\frac{\partial\left(\tau_{x y}\right)_{i}^{(6)}}{\partial y}+\left(\tau_{x z}\right)_{i}^{(5)}+\rho_{i} \frac{G_{x z}^{(5)}}{M}+\rho_{i} \frac{G_{x z}^{(3)}}{M}\left(U_{x}\right)_{i}^{(2)}=0 \\
\frac{\partial\left(\tau_{x y}\right)_{i}^{(6)}}{\partial x}+\frac{\partial\left(\tau_{y y}\right)_{i}^{(6)}}{\partial y}+\left(\tau_{y z}\right)_{i}^{(5)}+\rho_{i} \frac{G_{x z}^{(3)}}{M}\left(U_{y}\right)_{i}^{(2)}=0 \\
\frac{\partial\left(\tau_{z x}\right)_{i}^{(7)}}{\partial x}+\frac{\partial\left(\tau_{z y}\right)_{i}^{(7)}}{\partial y}+\left(\tau_{z z}\right)_{i}^{(6)}-\rho_{i} \frac{G_{x z}^{(5)}}{M}\left(x-c_{0}\right)+\rho_{i} \frac{G_{x z}^{(3)}}{M}\left(U_{z}\right)_{i}^{(3)}=0
\end{array}\right.
$$

где $G_{x z}^{(2 k-1)}$ - некоторые положительные константы; $M$-безразмерная масса поперечного сечения $\Omega$, приходящаяся на единицу длины.

Краевые условия на границе сечения для (9), (10),(11):

$$
\left(\tau_{\alpha x}\right)_{i}^{(2 k)} n_{x}+\left(\tau_{\alpha y}\right)_{i}^{(2 k)} n_{y}=0, \quad\left(\tau_{z x}\right)_{i}^{(2 k+1)} n_{x}+\left(\tau_{z y}\right)_{i}^{(2 k+1)} n_{y}=0, \quad \alpha \in(x, y) .
$$

Условия сопряжения между слоями $\Omega_{i}$ для (9), (10), (11):

$$
\begin{gathered}
\left(\tau_{n \beta}\right)_{i}^{(2 k)}=\left(\tau_{n \beta}\right)_{j}^{(2 k)}, \quad\left(\tau_{n z}\right)_{i}^{(2 k)}=\left(\tau_{n z}\right)_{j}^{(2 k)}, \\
\left(U_{\beta}\right)_{i}^{(2 k)}=\left(U_{\beta}\right)_{j}^{(2 k)}, \quad\left(U_{z}\right)_{i}^{(2 k+1)}=\left(U_{z}\right)_{j}^{(2 k+1)}, \quad \alpha \in(x, y, z), \beta \in(x, y) .
\end{gathered}
$$

Соотношения (9), (10), (11) вместе с (12), (13) образуют первые три вспомогательные краевые задачи для жесткостного номера $k=1,2,3$.

3.2. Асимптотическая выполнимость трехмерных уравнений теории упругости. Если формулы (4), (5) с учетом равенств (9)-(13) подставить в равенства полукраевой задачи (1), (2), то согласно [11] эти равенства будут выполняться асимптотически, т.е. невязки в выполнении этих равенств будут пропорциональны степеням малого параметра $\varepsilon$ :

$$
\begin{aligned}
& -\rho_{i} \frac{\partial^{2}\left(u_{\alpha}\right)_{i}}{\partial t^{2}}+\frac{\partial\left(\sigma_{\alpha x}\right)_{i}}{\partial x}+\frac{\partial\left(\sigma_{\alpha y}\right)_{i}}{\partial y}+\varepsilon \frac{\partial\left(\sigma_{\alpha z}\right)_{i}}{\partial z}=O\left(\varepsilon^{2 n+4}\right), \quad \alpha \in(x, y), \\
& -\rho_{i} \frac{\partial^{2}\left(u_{z}\right)_{i}}{\partial t^{2}}+\frac{\partial\left(\sigma_{x z}\right)_{i}}{\partial x}+\frac{\partial\left(\sigma_{y z}\right)_{i}}{\partial y}+\varepsilon \frac{\partial\left(\sigma_{z z}\right)_{i}}{\partial z}=O\left(\varepsilon^{2 n+3}\right) .
\end{aligned}
$$

Для уравнений движения (1a) невязка пропорциональна $\varepsilon^{(2 n+4)}$ при $\alpha=(x, y)$ и $\varepsilon^{(2 n+3)}$ при $\alpha=z$. Для равенств закона Гука (1b) невязка пропорциональна $\varepsilon^{(2 n+2)}$ при $(\alpha, \beta)=z$ и $\varepsilon^{(2 n+3)}$ при $\beta=z, \alpha=(x, y)$. Для остальных равенств закон Гука в каждом слое выполняется точно:

$$
\begin{gathered}
\left(\sigma_{\alpha z}\right)_{i}^{(n)}-\mu_{i}\left(e_{\alpha z}\right)_{i}^{(n)}=O\left(\varepsilon^{2 n+3}\right), \quad \alpha \in(x, y), \\
\left(\sigma_{z z}\right)_{i}^{(n)}-\left(\lambda_{i}+2 \mu_{i}\right)\left(e_{z z}\right)_{i}^{(n)}-\lambda_{i}\left(e_{x x}\right)_{i}^{(n)}-\lambda_{i}\left(e_{y y}\right)_{i}^{(n)}=O\left(\varepsilon^{2 n+2}\right) .
\end{gathered}
$$


Также точно выполняются равенства сопряжения слоев (1d) и условия на боковой поверхности (2). Окончательно можно сказать, что формулы (4)-(5) с учетом равенств (9)-(13) дают асимптотическое решение полукраевой задачи $(1),(2)$ с невязкой, пропорциональной $\varepsilon^{(2 n+2)}$. Таким образом, построенное асимптотическое решение является быстро убывающим по параметру $\varepsilon$, и в большинстве случаев достаточно ограничиться рассмотрением первых трех асимптотических приближений.

4. Уравнение свободных колебаний. Функция $u_{0}^{(n)}$ удовлетворяет уравнению свободных колебаний (6):

$$
\begin{gathered}
M \frac{\partial^{2} u_{0}^{(n)}}{\partial t^{2}}+\sum_{k=2}^{n+1} G_{x z}^{(2 k-1)} \frac{\partial^{2 k} u_{0}^{(n)}}{\partial z^{2 k}} \varepsilon^{2 k}=0 \\
I_{z z}^{(2 k)}=-\sum_{i=1}^{s} \int_{F_{i}}\left(x-c_{0}\right)\left(\tau_{z z}\right)_{i}^{(2 k)} d F, \quad G_{x z}^{(2 k-1)}=-\sum_{i=1}^{s} \int_{F_{i}}\left(\tau_{x z}\right)_{i}^{(2 k-1)} d F,
\end{gathered}
$$

где $G_{x z}^{(2 k-1)}$ - некоторые константы; $M$ - безразмерная масса поперечного сечения, приходящаяся на единицу длины.

Коэффициенты уравнения свободных колебаний (16) являются положительными числами. Уравнение имеет параболический тип, со степенью $2 n+2$ при старшей производной для асимптотического приближения с номером $n$. Дифференциальный оператор по пространтсвенной переменной в общем случае имеет $2 n+2$ независимых решения. В [11] показано, что для таких дифференциальных уравнений физическим смыслом обладают только 4 решения, которые являются регулярными возмущениями этого уравнения при $n=1$. Остальные решения являются сингулярными возмущениями и не обладают физическим смыслом. Поэтому для однозначного решения уравнения (16) достаточно 4 краевых условий на торцах (3). В случае шарнирного опирания условия примут вид

$$
\left\{\begin{array}{l}
u_{0}^{(n)}(z, \varepsilon)=0 \\
M_{y}^{(n)}(z, \varepsilon)=-\sum_{k=1}^{n} I_{z z}^{(2 k)} \frac{\partial^{2 k} u_{0}^{(n)}}{\partial z^{2 k}} \varepsilon^{2 k}=0, \quad z=0,1 .
\end{array}\right.
$$

Общее решение является линейной комбинацией счетного числа отдельных собственных гармоник. Уравнению свободных колебаний с краевыми условиями (17) удовлетворяют гармоники вида

$$
u_{0 m}^{(n)}=c_{m} \phi_{m}^{(n)} \cos \left(\omega_{m}^{(n)} t\right), \quad \phi_{m}=\sin (\pi m z),
$$

где $c_{m}$ - произвольные коэффициенты, $\phi_{m}^{(n)}(z, \varepsilon)$ - собственные моды колебаний. Собственные частоты $\omega_{m}^{(n)}$ поперечных колебаний определяются по формулам

$$
\begin{gathered}
\omega_{m}^{(n)}=\sqrt{\sum_{k=2}^{n+1} \frac{G_{x z}^{(2 k-1)}}{M}(\pi m \varepsilon)^{2 k}(-1)^{k}}, \\
\omega_{m}^{(1)}=(\pi m \varepsilon)^{2} \sqrt{\frac{G_{x z}^{(3)}}{M}}, \quad \omega_{m}^{(2)}=\omega_{m}^{(1)} \sqrt{1-\frac{G_{x z}^{(5)}}{G_{x z}^{(3)}}(\pi m \varepsilon)^{2}} .
\end{gathered}
$$

В силу того, что ряд в формуле (19) знакопеременный, для истинного значения собственной частоты $\omega_{m}$ справедливо следующее соотношение:

$$
\omega_{m}^{(2)}<\omega_{m}^{(4)}<\cdots<\omega_{m}<\cdots<\omega_{m}^{(3)}<\omega_{m}^{(1)} .
$$

Приближения образуют последовательность интервалов $\left[\omega_{m}^{(2 k)}, \omega_{m}^{(2 k-1)}\right]$, которая асимптотически сходится к истинной собственной частоте. Чтобы конечная сумма под корнем в формулах (19) 
сходилась, необходимо выполнение условия

$$
(\pi m \varepsilon)<1
$$

Условие (21) вносит ограничения на порядок собственной частоты и на величину малого параметра.

Первое приближение $\omega_{m}^{(1)}$ в формуле (19) полностью совпадает с формулой для частот по классической теории Бернулли-Эйлера для однородной балки и, по сути дела, является обобщением теории Бернулли-Эйлера на случай многослойных балок. Таким образом первое приближение собственных частот, полученное путем решения только первой краевой задачи для $k=1$, дает те же частоты, что и теория Бернулли-Эйлера. Первое приближение $\omega_{m}^{(2)}$ является уточнением первой и учитывает депланацию и инерцию вращения сечения. Формулы (19) позволяют определять собственные частоты колебаний для шарнирно опертых балок произвольного симметричного сечения, при этом изменение формы поперечного сечения влияет только на значения коэффициентов в уравнении колебаний $G_{x z}^{(2 k-1)}$. Таким образом задача о нахождении частот собственных колебаний сводится к нахождению величин $G_{x z}^{(2 k-1)}$ и решению вспомогательных краевых задач.

5. Аналитическое решение вспомогательных краевых задач. Рассмотрим случай, когда коэффициенты Пуассона $\nu_{i}$ в каждом слое $\Omega_{i}$ совпадают. Тогда краевая задача $(9),(12),(13)$ для $k=1$ имеет аналитическое решение для произвольной формы поперечного сечения:

$$
\begin{gathered}
\left(\tau_{z z}\right)_{i}^{(2)}=-\left(x-c_{0}\right) E_{i}, \quad\left(\tau_{\alpha \alpha}\right)_{i}^{(2)}=0, \quad \alpha \in(x, y), \\
\left(U_{x}\right)_{i}^{(2)}=\frac{1}{2}\left(-\nu y^{2}+\nu\left(x-c_{0}\right)^{2}+C_{2}\right), \quad \int_{F}\left(U_{x}\right)_{i}^{(2)} d F=0, \quad\left(U_{y}\right)_{i}^{(2)}=\nu y\left(x-c_{0}\right), \\
\int_{F_{i}}\left\langle\left(\tau_{z z}\right)_{i}^{(2)}\right\rangle d F=0 .
\end{gathered}
$$

Константа $c_{0}$ находится из условия $(23)$. Для $G_{x z}^{(3)}, G_{x z}^{(5)}$ с учетом $(22)$ справедливы формулы

$$
\begin{gathered}
G_{x z}^{(3)}=-\sum_{i=1}^{s} \int_{F_{i}}\left(\tau_{x z}\right)_{i}^{(3)} d F=-\sum_{i=1}^{s} \int_{F_{i}} x\left(\tau_{z z}\right)_{i}^{(2)} d F=[E I], \quad[E I]=\sum_{i=1}^{s} E_{i} I_{i}, \\
G_{x z}^{(5)}=-\sum_{i=1}^{s} \int_{F_{i}}\left(\tau_{x z}\right)_{i}^{(5)} d F=I_{z z}^{(4)}+\frac{G_{x z}^{(3)}}{M}[\rho I], \quad[\rho I]=\sum_{i=1}^{s} \rho_{i} I_{i}=\sum_{i=1}^{s} \int_{F_{i}} \rho_{i}\left(x-c_{o}\right)^{2} d F .
\end{gathered}
$$

Для нахождения остальных неизвестных воспользуемся процедурой усреднения жесткостных функций по сечению. Для тонкостенных балок искомые напряжения будут слабо меняться в пределах тонкой стенки и можно считать, что их средние величины будут почти совпадать с самими величинами. Подобная процедура решения краевых задач описана в [13].

Положим, что поперечное сечение имеет узкую прямоугольную форму. Введем операцию усреднения произвольной функции по сечению:

$$
\langle f\rangle=\frac{1}{b} \int_{-1 / 2 b}^{1 / 2 b} f(\alpha, s) d s .
$$

Для того, чтобы найти оставшиеся неизвестные жесткостные функции $\left(\tau_{z x}\right)_{i}^{(3)}$ и $\left(\tau_{z y}\right)_{i}^{(3)}$, применим операцию усреднения (25) ко второму уравнению (10). Воспользуемся условиями на боковой поверхности (12) и учтем решение (22):

$$
\frac{d\left\langle\left(\tau_{z x}\right)_{i}^{(3)}\right\rangle}{d x}=x E_{i}, \quad\left\langle\left(\tau_{z x}\right)_{i}^{(3)}\right\rangle=\int_{-1 / 2}^{x} E_{i} \xi d \xi .
$$


Функция $\left(\tau_{z y}\right)_{i}^{(3)}$ антисимметрична по ширине сечения, поэтому $\left(\tau_{z y}\right)_{i}^{(3)}=0$. Усредним второе равенство (7) и подставим в него усредненное третье выражение из (22), проинтегрируем по переменной $x$ и выразим усредненную жесткостную функцию $\left(U_{z}\right)_{i}^{(3)}$ :

$$
\left\langle\left(U_{z}\right)\right\rangle_{i}^{(3)}=\int_{-1 / 2}^{x}\left(\frac{\left\langle\left(\tau_{z x}\right)_{i}^{(3)}\right\rangle}{\mu_{i}}-\left\langle\left(U_{x}\right)_{i}^{(2)}\right\rangle\right) d \xi+C_{1}, \quad\left\langle\left(U_{x}\right)_{i}^{(2)}\right\rangle=\frac{1}{2} \nu\left(x^{2}-\frac{1}{12}\right) .
$$

Константа $C_{1}$, входящая в выражение $(27)$, находится из условия

$$
\int_{-1 / 2}^{1 / 2}\left\langle\left(\tau_{z z}\right)_{i}^{(4)}\right\rangle d x=0
$$

Выражение (28) следует из того, что при чистом изгибе продольное усилие равняется нулю.

Перейдем теперь ко второй краевой задаче. Усредним первое уравнение (10) и воспользуемся условиями на границе сечения (12):

Интегрируя (29), получим

$$
\frac{d\left(\left\langle\tau_{x x}\right)_{i}^{(4)}\right\rangle}{d x}+\left\langle\left(\tau_{z x}\right)_{i}^{(3)}\right\rangle+\frac{G_{x z}^{(3)}}{M}\left\langle\rho_{i}\right\rangle=0,\left.\quad\left\langle\left(\tau_{x x}\right)_{i}^{(4)}\right\rangle\right|_{x=-1 / 2}=0 .
$$

$$
\left\langle\left(\tau_{x x}\right)_{i}^{(4)}\right\rangle=-\int_{-1 / 2}^{x}\left\langle\left(\tau_{z x}\right)_{i}^{(3)}\right\rangle d \xi-\frac{G_{x z}^{(3)}}{M} \int_{-1 / 2}^{x}\left\langle\rho_{i}\right\rangle d \xi .
$$

В силу антисимметричности средние значения следующих функций равны нулю:

$$
\left\langle\left(\tau_{y y}\right)_{i}^{(4)}\right\rangle=0, \quad\left\langle\left(\tau_{x y}\right)_{i}^{(4)}\right\rangle=0
$$

Усредним по ширине сечения второе выражение (8):

$$
\left\langle\left(\tau_{z z}\right)_{i}^{(4)}\right\rangle=\nu\left\langle\left(\tau_{x x}\right)_{i}^{(4)}\right\rangle+E_{i}\left\langle\left(U_{z}\right)_{i}^{(3)}\right\rangle .
$$

Таким образом, величины $G_{x z}^{(3)}, G_{x z}^{(5)}$, необходимые для вычисления первых приближений собственных частот (19), можно найти аналитически по формулам (24), (26), (27), (28),(30), (32).

6. Численные расчеты. Для решения вспомогательных краевых задач были использованы два различных численных метода: метод коллокаций и наименьших квадратов (далее МКНК) и метод конечных элементов (далее МКЭ). Численные алгоритмы тестировались для случаев, когда аналитическое решение краевых задач (9)-(13) известно. Критерием правильности расчетов выступало требование совпадения двух независимых численных решений, полученных методами MKHK и MKЭ, а также выполнение необходимых условий разрешимости задач.

Для проверки правильности решения исходной задачи проведено сравнение с прямым трехмерным численным расчетом в конечно-элементном пакете COMSOL Multiphysics $\mathbb{R}$ (cм. www.comsol.com). В таблице 1 приведены значения первых двух собственных частот для трехслойной балки прямоугольного сечения, боковые слои которой выполнены из стали, а промежуточный слой выполнен из бетона. Параметры материалов задавались в безразмерном виде:

$$
\begin{gathered}
E_{1}=E_{3}=200, \quad E_{2}=25 ; \quad \nu_{1}=\nu_{3}=0,3, \quad \nu_{2}=0,2 ; \quad H_{1}=H_{3}=0,2, \quad H_{2}=0,6 ; \\
b=1, \quad L=10, \quad \rho_{1}=\rho_{3}=7850 \cdot 10^{-9}, \quad \rho_{2}=2300 \cdot 10^{-9},
\end{gathered}
$$

где $E_{i}, \nu_{i}, \rho_{i}, H_{i}$ - значение модуля Юнга, коэффициент Пуассона, плотность и высота $i$-го слоя соответственно; $b, L$ - ширина и длина балки соответственно. Первому асимптотическому приближению соответствуют значения, основанные на решении первой краевой задачи для жесткостного номера $k=1$. В этом случае не учитывается инерция вращения сечения и разница для первой собственной частоты составляет $8 \%$. Второе асимптотическое приближение является более точным и основано на решении первых двух краевых задач (9)-(13) для $k=1,2$. В этом случае разница для первой собственной частоты не превышает $1 \%$. Последний столбец соответствует 


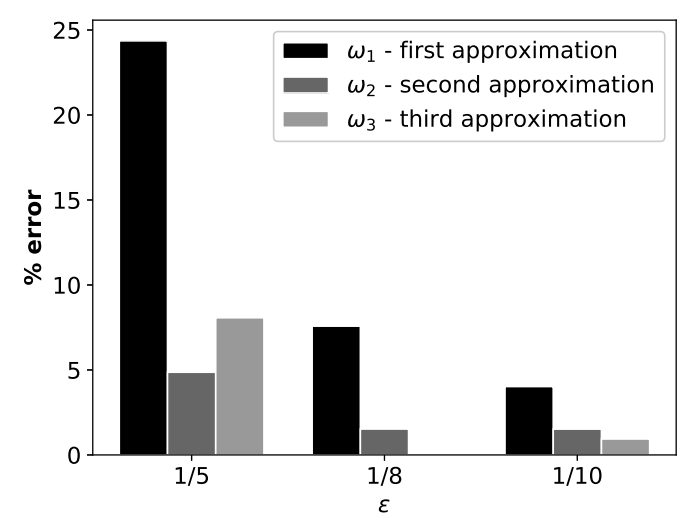

Рис. 2. Точность решения в зависимости от параметра $\varepsilon$

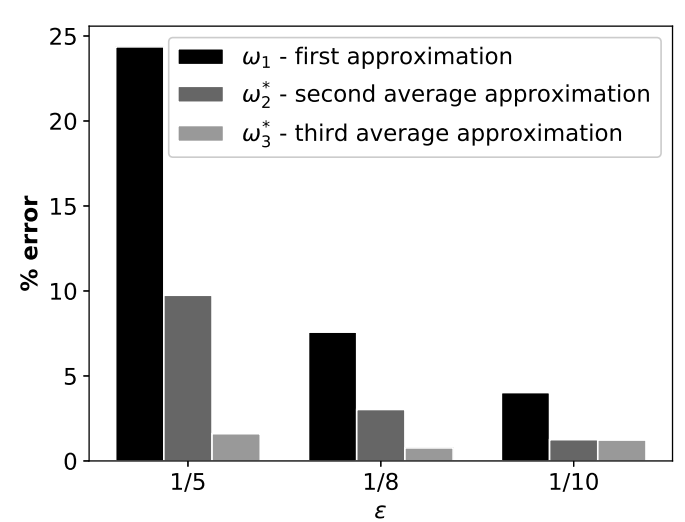

Рис. 3. Точность решения в зависимости от параметра $\varepsilon$ с использованием ускорения сходимости

первому асимптотическому приближению без учета разницы коэффициентов Пуассона. В этом случае поперечное сечение при изгибе остается плоским и верна гипотеза плоских сечений.

На основе полученных численных результатов можно сделать вывод, что учет разных коэффициентов Пуассона ведет к образованию особенностей у кромки слоев поперечного сечения. Схожий результат при решений краевых задач типа (9)-(13) был получен в [12] для задачи растяжения слоистого стержня. Как показывает практика зачастую наличием этих краевых эффектов можно пренебречь и влияние их на общее решение незначительно. В таблице 3 приведены значения для относительных разностей наиболеее значимых искомых величин: собственных частот, продольных напряжений и продольных перемещений. Знак $\Delta$ показывает относительную разницу между решением, когда коэффициенты Пуассона в слоях совпадают и решением, когда коэффициенты Пуассона отличаются. Из таблицы видно, что для расчета собственных частот наличием краевых эффектов можно полностью пренебречь.

В таблице 2 показана сходимость асимптотического решения для собственных частот в зависимости от параметра $\varepsilon$. Параметры материалов брались такими же, что и в (33), за исключением отношения длины балки к ее высоте. График (2) показывает относительную погрешность для первых трех асимптотических приближений. Точность решения для всех трех приближений растет с уменьшением параметра $\varepsilon$. Так как для последовательности асимптотических приближений верна формула (20), то для ускорения сходимости асимптотического решения целесообразно применить операцию, заключающуюся в усреднении предыдущего и текущего асимптотических приближений. Тогда для второго и третьего усредненных асимптотических приближений справедливы формулы

$$
\omega_{2}^{*}=\frac{\omega_{1}^{(1)}+\omega_{1}^{(2)}}{2}, \quad \omega_{3}^{*}=\frac{\omega_{1}^{(2)}+\omega_{1}^{(3)}}{2}
$$

На графике (3) отражена сходимость решения с использованием операции усреднения. В этом случае каждое следующее приближение точнее аппроксимирует искомую частоту. Важно отметить, что в случае коротких балок существенный вклад вносят краевые эффекты у торцов крепления балки, в то время как для длинных балок все асимптотические приближения дают хороший результат, в этом случае можно ограничиться первым приближением.

7. Заключение. Предложенный подход позволяет решать различные динамические задачи теории упругости, уравнения которых содержат малый параметр. Полученные асимптотические 
Таблица 1. Собственные частоты для трехслойной балки

\begin{tabular}{|c|c||c|c|c|}
\hline рад/c & $\begin{array}{c}\text { Comsol } \\
\text { Multiphysics }\end{array}$ & $\begin{array}{c}\text { 1 асимптотическое } \\
\text { приближение } \omega^{(1)}\end{array}$ & $\begin{array}{c}2 \text { асимптотическое } \\
\text { приближение } \omega^{(2)}\end{array}$ & $\begin{array}{c}\text { Гипотеза } \\
\text { плоских сечений }\end{array}$ \\
\hline$\omega_{1}$ & 158,53 & 170,52 & 158,93 & 170,67 \\
$\omega_{2}$ & 535,43 & 682,1 & 466,94 & 682,69 \\
\hline
\end{tabular}

Таблица 2. Сходимость частот в зависимости от параметра

\begin{tabular}{|c|c||c|c|c|}
\hline$\varepsilon$ & $\begin{array}{c}\text { Comsol } \\
\text { Multiphysics }\end{array}$ & $\begin{array}{c}\text { 1 асимптотическое } \\
\text { приближение } \omega^{(1)}\end{array}$ & $\begin{array}{c}2 \text { асимптотическое } \\
\text { приближение } \omega^{(2)}\end{array}$ & $\begin{array}{c}\text { 3 асимптотическое } \\
\text { приближение } \omega^{(3)}\end{array}$ \\
\hline $1 / 5$ & 512,61 & 637,49 & 487,67 & 553,98 \\
$1 / 8$ & 231,48 & 249,02 & 227,95 & 231,45 \\
$1 / 10$ & 153,21 & 159,37 & 150,88 & 151,77 \\
$1 / 15$ & 70,312 & 70,83 & 69,18 & 69,26 \\
\hline
\end{tabular}

Таблица 3. Относительные разности для собственных частот, продольных напряжений и продольных перемещений

\begin{tabular}{|c|c||c|c|c|}
\hline$E_{13}, E_{2}$ & $\nu_{13}, \nu_{2}$ & $\Delta \omega$ & $\Delta \tau_{z z}$ & $\Delta U_{z}$ \\
\hline$E_{13}=E_{2}=1$ & $\nu_{13}=\nu_{2}=0,33$ & 0,0 & 0,0 & 0,0 \\
$E_{13}=E_{2}=1$ & $\nu_{13}=0,33, \nu_{2}=0,25$ & 0,0001 & 0,011 & 0,17 \\
$E_{13}=E_{2}=1$ & $\nu_{13}=0,33, \nu_{2}=0,1$ & 0,0001 & 0,033 & 0,36 \\
$E_{13}=1, E_{2}=1$ & $\nu_{13}=0,4, \nu_{2}=0,05$ & 0,002 & 0,052 & 0,43 \\
$E_{13}=10, E_{2}=1$ & $\nu_{13}=0,33, \nu_{2}=0,25$ & 0,0026 & 0,011 & 0,077 \\
$E_{13}=100, E_{2}=1$ & $\nu_{13}=0,33, \nu_{2}=0,1$ & 0,0087 & 0,059 & 0,176 \\
\hline
\end{tabular}

решения являются быстро убывающими и в большинстве случаев достаточно ограничиться рассмотрением первых трех асимптотических приближений. Возникающие в процессе решения вспомогательные двумерные краевые задачи допускают аналитические решения в частных случаях, а в общем случае решаются численно.

Для задачи о поперечных колебаниях слоистой балки полученные асимптотические решения позволяют эффективно определять первые собственные частоты свободных колебаний. При этом решение на основе первого приближения совпадает с решением, полученным с использованием классической теории Бернулли-Эйлера для однородных балок, а все последующие приближения являются ее уточнением. Для существенно неоднородных балок, когда классическая теория Бернулли-Эйлера дает внушительную погрешность, использование второго и третьего асимптотических приближений является вполне обоснованым.

\section{СПИСОК ЛИТЕРАТУРЫ}

1. Агаловян Л. А. Асимптотическая теория анизотропных пластин и оболочек. - М.: Наука, 1997.

2. Амбариумян C. А. Общая теория анизотропных оболочек. - М.: Наука, 1974.

3. Андреев А. Н., Немировский Ю. В. Многослойные анизотропные оболочки и пластины: Изгиб, утойчивость, колебания. - Новосибирск: Наука, 2001.

4. Биргер И. А. Стержни, пластинки, оболчки. - М.: Ленанд, 2015.

5. Болотин В. В., Новичков Ю. Н. Механика многослойных конструкций. - М.: Машиностроение, 1980. 
6. Василъева А. Б., Бутузов В. Ф. Асимптотические разложения решений сингулярно возмущенных уравнений. - М.: Наука, 1973.

7. Голушко C. K., Немировский Ю. В. Прямые и обратные задачи механики упругих композитных пластин и оболочек вращения. - М.: Физматлит, 2008.

8. Голушко C. K., Идимешев C. В., Шапеев В. П. Метод коллокаций и наименьших невязок в приложении к задачам механики изотропных пластин// Вычисл. технол. - 2013. - 18, № 6. - С. 31-43.

9. Голушко C. K., Идимешев C. В., Шапеев В. П. Разработка и применение метода коллокаций и наименьших невязок к решению задач анизотропных слоистых пластин// Вычисл. технол. - 2014. - 19, № 5 . - C. $24-36$.

10. Голушко C. К., Идимешев С. В., Семисалов Б. В. Методы решения краевых задач механики композитных пластин и оболочек. - Новосибирск, 2014.

11. Горынин Г. Л., Немировский Ю. В. Пространственные задачи изгиба и кручения слоистых конструкций. Метод асимптотического расщепления. - Новосибирск: Наука, 2004.

12. Горынин Г. Л., Власко А. Ф. Исследование кромочного пограничного слоя в трехмерном стержне при растяжении // Доклю 3 й Всеросс. конф. «Проблемы оптимального проектирования сооружений» (Новосибирск, 15-17 апреля 2017 г.). - Новосибирск: НГАСУ, 2017. - С. 74-79.

13. Горынин Г. Л., Горънина О. Г. Исследование напряженно-деформируемого состояния трехслойного двутавра в пространственной постановке// Вестн. СибАДИ. - 2012. - № 5 (27). - С. 49-54.

14. Григолюк Э. И., Селезов И. Т. Неклассические теории колебаний стержней, пластин и оболочек// Итоги науки техн. Мех. тв. деформ. тел. - 1973. - 5.

15. Доннел Л. Г. Балки, пластины, оболочки. - М.: Наука, 1982.

16. Коул Дж. Методы возмущений в прикладной механике. - М.: Мир, 1972.

17. Образцов И. Ф., Нерубайло Б. В., Андрианов И. В. Асимптотические методы в строительной механике тонкостенных конструкций. - М.: Машиностроение, 1991.

18. Тимошенко С. П., Янг Д. Х., Уивер У. Колебания в инженерном деле. - М.: Машиностроение, 1985.

19. Alnaes M. S., Blechta J., Hake J., Johansson A., Kehlet B., Logg A., Richardson C., Ring J., Rognes M. E., Wells G. N. The FEniCS Project Ver. 1.5// Arch. Numer. Software. - 2015. - 3 .

20. Golushko S. K. Mathematical modeling and numerical optimization of composite structures// in: Optimum Composite Structures. — London: IntechOpen, 2019. - P. 13-34.

21. Gorynin G. L., Nemirovsky Yu. V. Tranverse vibration of laminated beams in three-dimensional formulation// Int. Appl. Mech. - 2005. - 41, № 6. - P. 631-645.

22. Shapeev V. P., Belyaev V. A., Golushko S. K., Idimeshev S. V. Possibilities and applications of the least squares collocation method// EPJ Web Conf. — 2018. — 173. — 01012.

Голушко Сергей Кузьмич

Новосибирский государственный университет;

Институт вычислительных технологий СО РАН, Новосибирск, Россия

E-mail: s.k.golushko@gmail.com

Горынин Глеб Леонидович

Сургутский государственный университет

E-mail: ggorynin@list.ru

Горынин Арсений Глебович

Новосибирский государственный университет

E-mail: arsgorynin@yandex.ru 